\title{
Characterizations of Distributions Based on Properties of Exponentially Shifted Records
}

\author{
M. Ahsanullah \\ Department of Management Sciences, Rider University, Lawrenceville, New Jersey, USA \\ ahsan@rider.edu \\ S.M. Ananjevskii \\ Department of Mathematics and Mechanics, St. Petersburg State University, St. Petersburg, Russia \\ V.B. Nevzorov \\ Department of Mathematics and Mechanics, St. Petersburg State University, St. Petersburg, Russia
}

Received 11 July 2015

Accepted 15 November 2015

1. Let $X_{1}, X_{2}, \ldots$ be a sequence of independent random variables (r.v.'s) with a common distribution function (d.f.) $F(x)$ and a probability density function (pdf) $f(x)$. Define the classical upper record times $L(n), n=1,2, \ldots$, and the classical upper record values $\mathrm{X}(1)<\mathrm{X}(2)<\ldots<\mathrm{X}(\mathrm{n})<\ldots$ as follows:

$$
\mathrm{L}(1)=1, \mathrm{~L}(\mathrm{n})=\min \left\{\mathrm{j}>\mathrm{L}(\mathrm{n}-1), \mathrm{X}_{\mathrm{j}}>\mathrm{X}_{\mathrm{L}(\mathrm{n}-1)}\right\}, \mathrm{n}=2,3, \ldots,
$$

and

In particular,

$$
X(n)=X_{L(n)}, \quad n=1,2, \ldots
$$

$$
\mathrm{X}(1)=\mathrm{X}_{1} \text {. }
$$

Let $\xi_{1}, \xi_{2}, \ldots$ be independent exponentially $\mathrm{E}(1)$-distributed r.v.'s with the probability density function $\mathrm{r}(\mathrm{x})=\exp (-\mathrm{x}), \mathrm{x} \geq 0$, and the distribution function $\mathrm{R}(\mathrm{x})=\max \{0,1-\exp (-\mathrm{x})\}$. The special case of record values based on r.v.'s $\xi_{1}, \xi_{2}, \ldots$ will be denoted as $\mathrm{Z}(1)<\mathrm{Z}(2)<\ldots<\mathrm{Z}(\mathrm{n})<\ldots$.

Record results in various spheres of human activities are very popular among citizens all over the world. It is enough to remember a lot of editions of the Guinness Book of Records. Therefore it is not surprising that there is the great interest among the specialists in probability and mathematical statistics to study and to solve the various problems which arise in the mathematical theory of records. There are a lot of monographs and papers (see, for example, references [1]-[9]), where the different aspects of the theory of records are considered. Meanwhile up to now there exist some of directions of this theory which attract the attention of mathematicians. One of these interesting directions is connected with obtaining the new characterizations of probability distributions based on the different properties of record values. Some of the corresponding results are given below.

2. One of the most important results in the theory of records is the representation of the exponential record values $Z(n), n=1,2, \ldots$, via the sums of independent $E(1)$-distributed r.v.'s (see, for example, Lecture 16 in [9]). 
Theorem 1. For any $n=1,2, \ldots$ the following equality in distribution is valid:

$$
\{Z(1), Z(2), \ldots, Z(n)\} \stackrel{\mathrm{d}}{=}\left\{\xi_{1}, \xi_{1}+\xi_{2}, \ldots, \xi_{1}+\xi_{2}+\ldots+\xi_{n}\right\} .
$$

It follows from (1) that the inter-record values $Z(1), Z(2)-Z(1), \ldots, Z(n)-Z(n-1), \ldots$ are independent and each of these differences has the standard exponential $\mathrm{E}(1)$-distribution.

Hence the following characterization of the exponential distribution ( see statement 1 and its proof in [7 ]) is not surprising. We propose below that the independent $\mathrm{E}(1)$-distributed random shifts $\xi_{1}$, $\xi_{2}$ and r.v.'s $\mathrm{X}_{1}, \mathrm{X}_{2}, \ldots$ are independent.

\section{Theorem 2. Relation}

$$
X(n-1)+\xi_{1} \stackrel{d}{=} X(n)
$$

holds for some arbitrary $n=2,3, \ldots$, if and only if

$$
F(x)=\max \{0,1-\exp (-(x-C))\}
$$

where $C$ is any constant.

It is naturally now to suppose that the analogous to (2) equality

$$
X(n-1) \stackrel{d}{=} X(n)-\xi_{2}
$$

also must be valid for the exponentially distributed X's. Hence the next characterization (see [7], statement 2) is surprising in some sense.

\section{Theorem 3. Relation}

$$
X(n-1) \stackrel{d}{=} X(n)-\xi_{2}
$$

holds for some arbitrary $n=2,3, \ldots$, if and only if

$$
F(x)=1-\exp \{-\exp (x-C) /(n-1)\}, \quad-\infty<x<\infty,
$$

where $C$ is any constant.

One can see that instead of the exponential distribution characterized by equality (2) relation (3), which is close to (2), characterizes the limiting for minimal order statistics type of distributions.

In [7] it was done the attempt to find in the simplest situation (for $n=2$ ) the general family of distributions which includes as the partial cases both the types of d.f.'s characterized by equalities (2) and (3). The following result was presented there. Note that the distributions characterized below in Theorem 4 are expressed via their inverse (quantile) functions

$$
\mathrm{Q}(\mathrm{x})=\inf \{\mathrm{y}: \mathrm{F}(\mathrm{y}) \geq \mathrm{x}\}, \quad 0<\mathrm{x}<1 .
$$

Theorem 4. Relation

$$
X(1)+a \xi_{1} \stackrel{\mathrm{d}}{=} X(2)-b \xi_{2},
$$

holds for any arbitrary $a$ and $b$, such that $a \geq 0, b \geq 0$ and $a+b>0$, if and only if 


$$
Q(x)=b \log (-\log (1-x))-\operatorname{alog}(1-x)+C, \quad 0<x<1,
$$

where $C$ is any constant.

Corollary 1. If $a=1, b=0$ in (5), then relation (6) has the form

$$
Q(x)=C-\log (1-x), \quad 0<x<1,
$$

and hence

$$
F(x)=1-\exp (-(x-C)) \text {, if } x \geq C \text {, and } F(x)=0 \text {, if } x<C \text {. }
$$

Thus the equality $X(1)+\xi_{1} \stackrel{d}{=} X(2)$ characterizes the exponential distribution with d.f.

$$
\mathrm{F}(\mathrm{x})=\max \{0,1-\exp (-(\mathrm{x}-\mathrm{C})) \text {. }
$$

Corollary 2. If $a=0, b=1$ in (5), then

$$
Q(x)=\log (-\log (1-x)), \quad 0<x<1,
$$

and hence

$$
F(x)=1-\exp (-\exp (x-C)), \quad-\infty<x<\infty .
$$

It confirms that the equality $\mathrm{X}(1)=\mathrm{X}(2)-\xi_{2}$ characterizes the limiting for minimal order statistics type of distributions.

3. It is interesting to solve the problem presented above for any $n=2,3, \ldots$ (not only for $n=2$ ). We prove here a more general result than the statement given in Theorem 4.

Consider now for arbitrary $n=2,3, \ldots$ relation

$$
\mathrm{X}(\mathrm{n}-1)+\mathrm{a} \xi_{1} \stackrel{\mathrm{d}}{=} \mathrm{X}(\mathrm{n})-\mathrm{b} \xi_{2},
$$

where, as above, exponentially E(1)-distributed r.v.'s $\xi_{1}, \xi_{2}$ and $X$ 's having some d.f. F(x) and pdf $f(x)$ are independent. It appears that the following generalization of Theorem 4 is valid.

Theorem 5. Relation

$$
X(n-1)+a \xi_{1} \stackrel{\mathrm{d}}{=} X(n)-b \xi_{2}
$$

holds for some $\mathrm{n}=2,3, \ldots$, and for any arbitrary $a$ and $b$, such that $a \geq 0, b \geq 0, a+b>0$, if and only if

$$
Q(x)=b(n-1) \log (-\log (1-x))-\operatorname{alog}(1-x)+C, \quad 0<x<1,
$$

where $C$ is any constant.

Remark 1. It is not difficult to see that the result of Theorem 4 presents the partial (under $n=2$ ) case of the more general Theorem 5 .

Corollary 3. If $a=1, b=0$ in (7), then (8) takes the form

$$
Q(x)=C-\log (1-x), \quad 0<x<1,
$$


and hence

$$
F(x)=1-\exp (-(x-C)) \text {, if } x \geq C \text {, and } F(x)=0 \text {, if } x<C \text {. }
$$

Thus the equality $X(n-1)+\xi_{1} \stackrel{d}{=} X(n)$ characterizes the exponential distribution with d.f.

$$
\mathrm{F}(\mathrm{x})=\max \{0,1-\exp (-(\mathrm{x}-\mathrm{C})) \text {. }
$$

Corollary 4. If $a=0, b=1$, then

$$
Q(x)=(n-1) \log (-\log (1-x))+C, \quad 0<x<1,
$$

and hence

$$
F(x)=1-\exp (-\exp (x-C) /(n-1)), \quad-\infty<x<\infty .
$$

We can present separately one more partial case of Theorem 5 .

Corollary 5. If $a=1, b=1$, then relation

$$
X(n-1)+\xi_{1} \stackrel{\mathrm{d}}{=} X(n)-\xi_{2}
$$

holds if and only if

$$
Q(x)=(n-1) \log (-\log (1-x))-\log (1-x)+C, \quad 0<x<1 .
$$

Note that equality (10) can be rewritten in the following form:

$$
(\mathrm{n}-1) \log (-\log (1-\mathrm{F}(\mathrm{x})))-\log (1-\mathrm{F}(\mathrm{x}))=\mathrm{x}-\mathrm{C}, \quad-\infty<\mathrm{x}<\infty,
$$

where $\mathrm{C}$ is any constant.

\section{Proof of Theorem 5.}

Let $F_{n}(x)$ and $f_{n}(x), n=1,2, \ldots$, denote correspondingly d.f.'s and pdf's of record values $X(1), X(2), \ldots$ It is known (see, for example, [2], [5] or [8]) that

$$
\begin{gathered}
\mathrm{F}_{\mathrm{n}}(\mathrm{x})=\frac{1}{(n-1) !} \int_{0}^{-\log (1-F(x))} v^{n-1} \exp (-v) d v= \\
1-(1-\mathrm{F}(\mathrm{x})) \sum_{k=0}^{n-1}(-\log (1-F(x)))^{k} / k ! \quad, \quad \mathrm{n}=1,2, \ldots,
\end{gathered}
$$

and

$$
f_{n}(x)=f(x)(-\log (1-F(x)))^{n-1} /(n-1) !, \quad n=1,2, \ldots
$$

Note also that

$$
F_{n-1}(x)-F_{n}(x)=(1-F(x))(-\log (1-F(x)))^{n-1} /(n-1) !, \quad n=2,3, \ldots .
$$

It is not difficult to notice that if $a \neq 0$ and $b \neq 0$ then 


$$
\mathrm{P}\left\{\mathrm{X}(\mathrm{n})-\mathrm{b} \xi_{2}<\mathrm{x}\right\}=\frac{1}{b} \exp \left(\frac{x}{b}\right) \int_{x}^{\infty} \exp (-v / b) F_{n}(v) d v
$$

and

$$
\mathrm{P}\left\{\mathrm{X}(\mathrm{n}-1)+\mathrm{a} \xi_{1}<\mathrm{X}\right\}=\frac{1}{a} \exp \left(-\frac{x}{a}\right) \int_{-\infty}^{x} \exp (v / a) F_{n-1}(v) d v .
$$

Thus we get from (15) and (16) that relation (7) holds if

$$
\int_{x}^{\infty} \exp (-v / b) F_{n}(v) d v=\frac{b}{a} \exp \left(-x\left(\frac{1}{a}+\frac{1}{b}\right) \int_{-\infty}^{x} \exp (v / a) F_{n-1}(v) d v .\right.
$$

After differentiating the both parts of (17) one obtains easily that

$$
\exp (\mathrm{x} / \mathrm{a}) \mathrm{F}_{\mathrm{n}}(\mathrm{x})=\frac{a+b}{a^{2}} \int_{-\infty}^{x} \exp (v / a) F_{n-1}(v) d v-\frac{b}{a} \exp (x / a) F_{n-1}(x) .
$$

One more differentiating allows us to find that

$$
\begin{gathered}
\frac{1}{a} \exp (\mathrm{x} / \mathrm{a}) \mathrm{F}_{\mathrm{n}}(\mathrm{x})+\exp (\mathrm{x} / \mathrm{a}) \mathrm{f}_{\mathrm{n}}(\mathrm{x})= \\
\frac{a+b}{a^{2}} \exp (\mathrm{x} / \mathrm{a}) \mathrm{F}_{\mathrm{n}-1}(\mathrm{x})-\frac{b}{a^{2}} \exp (\mathrm{x} / \mathrm{a}) \mathrm{F}_{\mathrm{n}-1}(\mathrm{x})-\frac{b}{a} \exp (\mathrm{x} / \mathrm{a}) \mathrm{f}_{\mathrm{n}-1}(\mathrm{x})
\end{gathered}
$$

and hence

$$
\frac{1}{a}\left(\mathrm{~F}_{\mathrm{n}-1}(\mathrm{x})-\mathrm{F}_{\mathrm{n}}(\mathrm{x})\right)=\mathrm{f}_{\mathrm{n}}(\mathrm{x})+\frac{b}{a} \mathrm{f}_{\mathrm{n}-1}(\mathrm{x}) .
$$

Recalling relations (13) (for $\mathrm{n}$ and $\mathrm{n}-1$ ) and (14), after some evident transformations one gets from equality (18) that

$$
(1-\mathrm{F}(\mathrm{x}))(-\log (1-\mathrm{F}(\mathrm{x})))=\mathrm{af}(\mathrm{x})(-\log (1-\mathrm{F}(\mathrm{x})))+\mathrm{b}(\mathrm{n}-1) \mathrm{f}(\mathrm{x}) .
$$

Indeed, it was proved that (19) is valid if $a>0$ and $b>0$. It is not difficult to check that if $a=0$ and $b>0$, then, considering relation

$$
\mathrm{F}_{\mathrm{n}-1}(\mathrm{x})=\frac{1}{b} \exp \left(\frac{x}{b}\right) \int_{x}^{\infty} \exp (-v / b) F_{n}(v) d v,
$$

we also easily get equality (19) with $\mathrm{a}=0$.

If $\mathrm{a}>0$ and $\mathrm{b}=0$ then we deal with equality

$$
\mathrm{F}_{\mathrm{n}}(\mathrm{x})=\frac{1}{a} \exp \left(-\frac{x}{a}\right) \int_{-\infty}^{x} \exp (v / a) F_{n-1}(v) d v
$$

and also can get that in this case equality (19) is also valid with $b=0$.

Thus relation (19) holds in all situations when $a \geq 0, b \geq 0$ and $a+b>0$. It follows immediately from (19) that

$$
1=\operatorname{af}(\mathrm{x}) /(1-\mathrm{F}(\mathrm{x}))+\mathrm{b}(\mathrm{n}-1) \mathrm{f}(\mathrm{x}) /(1-\mathrm{F}(\mathrm{x}))(-\log (1-\mathrm{F}(\mathrm{x}))) .
$$

Recalling that $(-\mathrm{f}(\mathrm{x}))$ is the derivative of $(1-\mathrm{F}(\mathrm{x}))$, it is possible to consider (21) as the corresponding differential equation and to get the solution of it in the following form: 


$$
x-C=-a \log (1-F(x))+b(n-1) \log (-\log (1-F(x))),
$$

where $\mathrm{C}$ is any constant.

Substituting to (22) the quantile function $\mathrm{Q}(\mathrm{x})$ instead of $\mathrm{x}$ one obtains finally that

$$
\mathrm{Q}(\mathrm{x})=\mathrm{C}+\mathrm{b}(\mathrm{n}-1) \log (-\log (1-\mathrm{x}))-\mathrm{a} \log (1-\mathrm{x}), \quad 0<\mathrm{x}<1,
$$

and this coincides with the statement of Theorem 5 .

Remark 2. The standard method allows reformulating easily Theorem 5 for the case when one is going to investigate the lower records instead of upper record values. It is enough to consider r.v.'s $Y_{k}=-X_{k}, k=1,2, \ldots$ Then the corresponding lower record values $y(1)>y(2)>\ldots>y(n)>\ldots$, based on $Y$ 's, will coincide with values $\mathrm{X}(1),-\mathrm{X}(2), \ldots,-\mathrm{X}(\mathrm{n}), \ldots$.

Taking into account that in this situation

$$
\mathrm{H}(\mathrm{x})=\mathrm{P}\left\{\mathrm{Y}_{\mathrm{k}}<\mathrm{x}\right\}=1-\mathrm{F}(-\mathrm{x}), \mathrm{k}=1,2, \ldots,
$$

and the inverse function (denote it $\mathrm{R}(\mathrm{x})$ ) for the d.f. $\mathrm{H}(\mathrm{x})$ is expressed as

$$
\mathrm{R}(\mathrm{x})=-\mathrm{Q}(1-\mathrm{x}), \quad 0<\mathrm{x}<1,
$$

one can reformulate Theorem 5 for the lower record values.

\section{Theorem 6. Relation}

$$
y(n-1)-a \xi_{1} \stackrel{\mathrm{d}}{=} y(n)+b \xi_{2},
$$

holds for some $n=2,3, \ldots$, and for any arbitrary $a$ and $b$, such that $a \geq 0, b \geq 0, a+b>0$, if and only if

$$
R(x)=-b(n-1) \log (-\log (1-x))+\operatorname{alog}(1-x)+C, \quad 0<x<1
$$

where $C$ is any constant.

Two partial cases of Theorem 6 can be mentioned here. The next results are analogous to statements presented above in Corollaries 3 and 4.

\section{Corollary 6. Relation}

$$
y(n-1)-\xi_{1}=y(n), \quad n=2,3, \ldots,
$$

holds if and only if

$$
R(x)=\log (x)+C, \quad 0<x<1,
$$

and hence

$$
H(x)=\exp (x-C) \text {, if } x \leq C \text {, and } H(x)=1 \text {, if } x>C,
$$

where $C$ is any constant.

Thus the equality $y(n-1)-\xi_{1}=y(n)$ characterizes the family of the negative exponential distributions.

\section{Corollary 7. Relation}

$$
y(n-1) \stackrel{\mathrm{d}}{=} y(n)+\xi_{2}, \quad n=2,3, \ldots,
$$

holds if and only if 


$$
R(x)=C-(n-1) \log (-\log x), \quad 0<x<1,
$$

and hence

$$
H(x)=\exp (-\exp (-(x-C) /(n-1))), \quad-\infty<x<\infty,
$$

where $C$ is any constant.

Thus equality (23) characterizes the family of the extreme value distributions, which are also named sometimes as log-Weibull or doubly exponential distributions.

\section{References}

[1] M. Ahsanullah, Record Statistics. (Nova Science Publishers Inc., New York, NY, 1995).

[2] M. Ahsanullah, Record Values-Theory and Applications. (University Press of America, Lanham, MD, 2004).

[3] M. Ahsanullah and V.B. Nevzorov, Ordered Random Variables. (Nova Science Publishers Inc., New York, 2001).

[4] M. Ahsanullah and V.B. Nevzorov, Record Statistics, International Encyclopedia of Statistical Science, part 18 (2011) 1195-1202.

[5] M. Ahsanullah and V.B. Nevzorov, Records via Probability Theory. (Atlantis Press, 2015).

[6] M. Ahsanullah, V.B. Nevzorov and L.N. Nevzorova, Characterizations of distributions via record values with random exponential shifts. J. Statistical Theory and Application 13(4), (2014) 311-316.

[7] M. Ahsanullah, V.B. Nevzorov and L.N. Nevzorova, Characterizations of distributions via record values with random exponential shifts II. J. of Applied Statistical Science 21(2), (2015) 157-165.

[8] B.C. Arnold, N. Balakrishnan and H.N. Nagaraja, Records (John Wiley \& Inc., New York, 1997).

[9] V.B. Nevzorov, Records: Mathematical Theory. (American Math. Soc., Providence, Rhode Island, 2001). 